\title{
El uso sostenible del agua: Tributos medioambientales y nuevos procesos de descontaminación mediante energías renovables"
}

\author{
ISABEL MARÍA ROMÁN SÁNCHEZ \\ Departamento de Economía Aplicada, UNIVERSIDAD DE ALMERÍA, ESPAÑA. E-mail: \\ iroman@ual.es
}

IRENE CARRA

Departamento de Ingeniería Química, UNIVERSIDAD DE ALMERÍA, ESPAÑA. E-mail: irenecarra@ual.es

JOSÉ ANTONIO SÁNCHEZ PÉREZ

Departamento de Ingeniería Química, UNIVERSIDAD DE ALMERÍA, ESPAÑA. E-mail: jsanchez@ual.es

\section{RESUMEN}

La presencia de contaminación persistente y tóxica en las aguas ha impulsado el desarrollo de nuevas tecnologías de tratamiento, como es el caso, de la fotocatálisis solar homogénea, foto-Fenton solar, que aprovecha esa fuente de energía renovable para disminuir los costes del proceso. El fallo de mercado que supone la contaminación originada por el vertido de aguas residuales industriales, se corrige con el establecimiento de tributos medioambientales. La heterogeneidad en el diseño de estos tributos afecta tanto a los parámetros de contaminación considerados como a su peso específico en el cálculo de la cuota a pagar. En este trabajo se discute la posibilidad de los cánones de saneamiento como correctores de este fallo de mercado y como incentivos para invertir en tecnologías y procesos de depuración sostenibles.

Palabras clave: Agua, Energía Solar, Canon de Saneamiento, Externalidades.

\section{The Sustainable Use of Water: Environmental Taxes and New Decontamination Processes Using Renewable Energy}

\begin{abstract}
The presence of persistent and toxic pollutants in waters has encouraged the development of new water treatment technologies, such as the solar photocatalysis process, solar photo-Fenton, which uses the Sun as renewable source of energy to diminish process costs. The market failure associated to the pollution originated by industrial wastewater discharges is corrected with the establishment of environmental taxes. The heterogeneity in the design of these taxes affects both the considered pollution parameters and their specific weight in the calculation of the tax to be paid. In this paper, it is discussed the capability of the environmental taxes to correct the water pollution as market failure, and as an incentive to invest in sustainable technologies and water treatment processes.
\end{abstract}

Keywords: Water, Solar Energy, Environmental Tax, Externalities.

Clasificación JEL: H23, Q25, Q53, Q55, Q56

\footnotetext{
* Los autores desean agradecer la financiación recibida al Ministerio de Economía y Competitividad y FEDER (Proyecto CTQ 201020740-C03-01/PPQ). Irene Carra quiere agradecer al Ministerio de Educación, Cultura y Deporte su beca FPU (FPU2010-3218).
}

Artículo recibido en febrero de 2013 y aceptado en abril de 2013

Artículo disponible en versión electrónica en la página www.revista-eea.net, ref. ə-31114 


\section{INTRODUCCIÓN}

La Directiva Marco del Agua, DMA en adelante, (Directiva 2000/60/CE) ha supuesto un gran impulso en las políticas sobre protección de aguas y reducción de la contaminación en los estados miembros. El artículo 4 de dicha Directiva trata los objetivos medioambientales de las aguas superficiales y de la necesidad de aplicar las medidas que reduzcan progresivamente la contaminación, así como reducir o interrumpir gradualmente los vertidos. La preocupación por el cuidado del medioambiente va creciendo en la sociedad, impulsando a los gobiernos a tomar medidas que permitan la conservación de los espacios naturales, la mejora del entorno ambiental y la reducción de la contaminación (Jiménez y Lafuente, 2007).

Con el transcurso del tiempo aumenta la conciencia de que el agua, como bien escaso, tiene valor económico, de manera que la gestión del agua, en su condición de bien económico, es un medio importante para conseguir un aprovechamiento eficaz y equitativo, y favorecer la conservación y protección de los recursos hídricos (Beamonte, 2010).

Dentro del análisis económico, se pone énfasis al papel que deben cumplir los precios del agua como incentivos adecuados para el uso eficiente del agua y consecución de los objetivos ambientales (Del Villar, 2010).

La contaminación se define, desde el punto de vista ecológico, como la alteración de las propiedades de un medio por la incorporación, generalmente debida a la acción directa o indirecta del hombre, de perturbaciones o materiales que introducen modificaciones de la estructura y la función de los ecosistemas afectados. Sin embargo, la definición económica de la contaminación depende tanto de los efectos ocasionados sobre el medio ambiente (por ejemplo, extinción de especies, sobreexplotación de ríos y de acuíferos, perjuicios sobre la salud pública y aparición de epidemias, etc.) como de la reacción humana frente a dichos efectos que, en general, se concreta en alguna forma de pérdida de bienestar.

El concepto económico de contaminación es un ejemplo de externalidad negativa pues la actividad del contaminador provoca una pérdida de bienestar a otros agentes que no reciben una compensación por ello. Asimismo, la contaminación entendida como fallo del mercado, supone un problema económico, además de ambiental, que justifica la intervención pública correctora.

Para internalizar los efectos externos negativos que generan los agentes económicos, la intervención pública puede llevarse a cabo mediante:

a) controles directos o regulaciones convencionales

b) instrumentos de mercado (impuestos y derechos transferibles de contaminación).

Los instrumentos de mercado generan un estímulo tecnológico permanente, 
puesto que definen un sistema de premios/pagos del que se pueden derivar beneficios/ahorros si los agentes introducen conductas reductoras de la contaminación. Así, cada agente contaminador adapta su conducta para pagar por contaminar o por el contrario, para invertir en tecnologías que le permitan contaminar menos con miras a pagar lo mínimo posible en la cuota de estos tributos.

Y, puesto que el problema de la contaminación puede abordarse fundamentalmente a través de un cambio tecnológico que permita la adopción de los procesos productivos no contaminantes, puede ser recomendable dirigir parte de la recaudación ambiental conseguida, mediante una reforma fiscal verde, a la subvención al desarrollo y difusión de nuevas tecnologías. Sin olvidar que la tributación ambiental genera por sí misma incentivos al abandono de procesos contaminantes y propensión a la inversión en tecnologías limpias (Labandeira, 2010).

En línea con el planteamiento propuesto por la DMA para reducir la contaminación de las aguas superficiales, en el presente trabajo tratamos de exponer el papel que juegan los cánones de saneamiento como instrumentos que incentivan la inversión en nuevas tecnologías de tratamiento de aguas residuales. En concreto, hemos considerado los tratamientos de oxidación avanzada que utilizan energías renovables (fotocatálisis solar).

En este sentido, en la Sección 2 estudiamos las estructuras de las tarifas de los cánones de saneamiento y analizamos los parámetros físico-químicos de contaminación que contemplan para penalizar al agente contaminador por el vertido de efluentes. En la Sección 3 se describe un proceso de descontaminación de aguas residuales industriales mediante energías renovables, presentando los costes del mismo en la Sección 4. Finalmente, en la Sección 5 analizamos la rentabilidad de invertir o no, en estas tecnologías, en función del importe de los cánones de saneamiento.

\section{CANONES DE SANEAMIENTO}

En cumplimiento del artículo 4 de la DMA, los países miembros han desarrollado tributos medioambientales que gravan los vertidos al agua. Son conocidos como cánones de saneamiento y en cada país tienen un diseño específico.

Los cánones de saneamiento están destinados a financiar los procesos de tratamiento de agua residual, tanto la inversión de las instalaciones de depuración como los costes de operación y de mantenimiento. En Europa este tributo suele ser de competencia local, como es el caso de España, cuya competencia es autonómica.

En materia de aguas, las Comunidades Autónomas intervienen -además de en la fijación de las tarifas de distribución y consumo del agua potable- en la 
fijación de las tasas relacionadas con la depuración de aguas residuales (Beamonte, 2010).

En nuestro país en materia de tributos que gravan los vertidos al agua, nos encontramos con aquellos de competencia estatal, regulados por la ley de Aguas y la ley de Costas y los de competencia autonómica, regulados por la ley de cada comunidad.

A nivel estatal, ambos cánones de vertido (Ley de Aguas y Ley de Costas), se identifican con la categoría de tasas, así el artículo 113.1 de la Ley de Aguas, dice que "los vertidos al dominio público hidráulico estarán gravados con una tasa destinada al estudio, control, protección y mejora del medio receptor de cada cuenca hidrográfica, que se denominará canon de control de vertidos." Los sujetos pasivos son aquellos que realicen el hecho imponible, es decir, producción/vertido de agua residual. El importe del canon de control de vertidos será el producto del volumen de vertido autorizado por el precio unitario de control de vertido. Este precio unitario se calculará multiplicando el precio básico por metro cúbico por un coeficiente de mayoración o minoración, que se establecerá reglamentariamente en función de la naturaleza, características y grado de contaminación del vertido, así como por la mayor calidad ambiental del medio físico en que se vierte.

A nivel autonómico, cada comunidad diseña su canon de saneamiento. Como resultado existe una gran variación tanto en la estructura como en los parámetros de contaminación considerados en cada normativa, lo que conlleva marcadas diferencias en el importe de la cuota a pagar por este impuesto. De las diecisiete comunidades autónomas (CCAA) que hay en España, sólo diez tienen regulado el canon de saneamiento (ver Tabla 1).

La estructura de la tarifa del canon tiene dos términos, el primero es un coste fijo, independiente del volumen vertido y el segundo un término variable que depende del volumen de agua residual vertido y de su nivel de contaminación, es decir, de la carga contaminante. Para caracterizar la contaminación del agua vertida se utilizan distintos parámetros, como la demanda química de oxígeno (DQO), la presencia de sólidos en suspensión (SS), que se tienen en cuenta en los cánones de las diez comunidades autónomas, la conductividad (C), la demanda biológica de oxígeno (DBO), la concentración de nutrientes, principalmente nitrógeno $(\mathrm{N})$ y fósforo $(\mathrm{P})$, metales pesados (MP), sustancias inhibitorias (SI) y la diferencia de temperatura $(\Delta \mathrm{T})$. Destacamos que en las diez comunidades autónomas en las ecuaciones para calcular la cuota, se tienen en cuenta la DQO y los SS. Sin embargo, el resto de parámetros son tenidos en cuenta en algunas comunidades y en otras no. Algunos de estos parámetros se incluyen en el diseño del canon teniendo en cuenta principalmente el tipo de industrias predominantes en la región. Por ejemplo, en el caso de Asturias se incluye la concentración de metales pesados debido a la presencia de la indus- 
tria minera. Por otro lado, en algunos casos para establecer la cuota del canon se toma como referencia el nivel de contaminación del agua residual doméstica, como ocurre en de Navarra, Valencia, La Rioja y Murcia.

\section{Tabla 1}

Cánones de saneamiento de las diez comunidades autónomas estudiadas

\begin{tabular}{|c|c|}
\hline CC AA & Cuota del canon de saneamiento \\
\hline $\begin{array}{c}\text { Aragón } \\
\text { (Ley 6/2001) }\end{array}$ & $\begin{array}{l}\mathrm{CS}_{\text {Aragón }}(€ / \text { /año })=\mathrm{CF}+\mathrm{CV} \cdot \mathrm{Q}_{\mathrm{w}} \\
\mathrm{CF}(€ / \text { año })=194,75 \\
\mathrm{CV}\left(€ / \mathrm{m}^{3}\right)=0,396 \cdot \mathrm{SS}+0,551 \cdot \mathrm{DQO}+1,082 \cdot \mathrm{N}+4,453 \cdot \mathrm{C}+0,00541 \cdot \mathrm{MP}+0,01286 \cdot \mathrm{SI}\end{array}$ \\
\hline $\begin{array}{c}\text { Asturias } \\
\text { (Ley 1/1994) }\end{array}$ & $\begin{array}{l}\mathrm{CS}_{\text {Asturias }}(€ / \text { año })=\mathrm{CF}+\mathrm{CV} \cdot \mathrm{Q}_{\mathrm{w}} \\
\mathrm{CF}(€ / \text { año })=60 \\
\mathrm{CV}\left(€ / \mathrm{m}^{3}\right)=0,12+0,4673 \cdot \mathrm{SS}+0,4154 \cdot \mathrm{DQO}+2,3814 \cdot \mathrm{N}+4,3416 \cdot \mathrm{P}+0,5247 \cdot \mathrm{C}+0,004 \cdot \Delta \mathrm{T}+ \\
0,0239 \cdot \mathrm{MP}+0,0072 \cdot \mathrm{SI}\end{array}$ \\
\hline $\begin{array}{c}\text { Cantabria } \\
\text { (Ley } 2 / 2002 \text { ) }\end{array}$ & $\begin{array}{l}\mathrm{CS}_{\text {Cantabria }}(€ / \mathrm{año})=\mathrm{CF}+\mathrm{CV} \cdot \mathrm{Q}_{\mathrm{w}} \\
\mathrm{CF}(€ / \mathrm{año})=14,88 \\
\mathrm{CV}\left(€ / \mathrm{m}^{3}\right)=0,2316 \cdot \mathrm{SS} \cdot \mathrm{C}_{\mathrm{pSS}}+0,2681 \cdot \mathrm{DQO} \cdot \mathrm{C}_{\mathrm{pDQO}}+0,2925 \cdot \mathrm{N} \cdot \mathrm{C}_{\mathrm{pN}}+0,5850 \cdot \mathrm{P} \cdot \mathrm{C}_{\mathrm{pP}}+ \\
3,6683 \cdot \mathrm{C} \cdot \mathrm{C}_{\mathrm{pC}}+0,004595 \cdot \mathrm{SI} \cdot \mathrm{C}_{\mathrm{pSI}}+0,000048 \cdot \Delta \mathrm{T} \cdot \mathrm{C}_{\mathrm{p} \Delta \mathrm{T}} \\
\mathrm{C}_{\mathrm{pSS}}, \mathrm{C}_{\mathrm{pDQO}}, \mathrm{C}_{\mathrm{pN}}, \mathrm{C}_{\mathrm{pP}}, \mathrm{C} \mathrm{C}_{\mathrm{pC}}, \mathrm{C}_{\mathrm{pSI}}, \mathrm{C}_{\mathrm{p} \Delta \mathrm{T}} \text { : coeficientes pico para cada parámetro, definidos } \\
\text { como la relación entre la concentración máxima y media; se ha considerado un valor de } 1 \\
\text { para todos los parámetros. }\end{array}$ \\
\hline $\begin{array}{c}\text { Cataluña } \\
\text { (Ley } 3 / 2003)\end{array}$ & $\begin{array}{l}C S_{\text {Cataluña }}(€ / \text { año })=Q_{c} \cdot(0,134+C C) \\
C C\left(€ / m^{3}\right)=[0,406 \cdot S S+0,8122 \cdot D Q O+0,6166 \cdot N+1,2334 \cdot P+6,4979 \cdot C+ \\
0,00963 \cdot S I] C_{p} \cdot C_{v} \cdot C_{w} \cdot C_{d} \cdot C_{s} \cdot C_{r} \\
C_{p}: \text { coeficiente pico, razón entre máximo y la media (se ha considerado 1); } \\
\left.C_{w} \text { : coeficiente de descarga (se ha considerado } 1.5\right) ; C_{d}: \text { coeficiente de dilución (descargas } \\
\text { al mar, no aplicable para este caso); } C_{s}: \text { coeficiente de salinidad (se ha considerado 1); } C_{r}: \\
\text { coeficiente de reutilización; si se consume agua regenerada (no aplicable para este caso). }\end{array}$ \\
\hline $\begin{array}{c}\text { Galicia } \\
\text { (Ley } 9 / 2010 \text { ) }\end{array}$ & $\begin{array}{l}C S_{\text {Galicia }}(€ / \text { año })=C F+C V \cdot Q_{w} \\
C F(€ / \text { año })=30 \\
C V(€ / \text { año })=0,246 \cdot S S+0,493 \cdot D Q O+0,369 \cdot \mathrm{N}+0,74 \cdot P+3,955 \cdot C+0,0111 \cdot \mathrm{MP}+0,052 \cdot S I\end{array}$ \\
\hline $\begin{array}{c}\text { La Rioja } \\
\text { (Ley } 5 / 2000)\end{array}$ & $\begin{array}{l}C S_{\text {Rioja }}(€ / a n ̃ o)=0,35 \cdot C C \cdot Q_{w} \\
C C=0,276 \cdot S S / 0,22+0,458 \cdot D Q O / 0,5+0,266 \cdot C / 0,0024 \\
C C_{\text {min }}=0,35\end{array}$ \\
\hline $\begin{array}{c}\text { Madrid } \\
\text { (Ley 10/1993) }\end{array}$ & $\begin{array}{l}\mathrm{CS}_{\text {Madrid }}(€ / \text { año })=\mathrm{TS}+\mathrm{CV} \cdot \mathrm{Q}_{\mathrm{C}} \\
\mathrm{TS}(€ / \text { año })=1,254 \cdot\left(\varnothing^{2}+5 \cdot \varnothing\right) ; \text { siendo } \varnothing(\mathrm{m})=0,2 \\
\mathrm{CV}\left(€ / \mathrm{m}^{3}\right)=0,5104 \cdot \mathrm{CC} \\
\mathrm{CC}=1,1 \cdot \mathrm{SS}+\mathrm{DQO}+1,65 \cdot \mathrm{DBO} \\
\mathrm{CC}_{\min }=1\end{array}$ \\
\hline $\begin{array}{c}\text { Murcia } \\
\text { (Ley } 3 / 2000 \text { ) }\end{array}$ & $\begin{array}{l}\operatorname{CS}_{\text {Murcia }}(€ / \text { año })=\left(T S+\left(0,34 \cdot Q_{C}\right)\right) \cdot C C \cdot C_{v} \\
\text { TS }(€ / \text { año })=30 \\
C C=(S S / 0,3+2 \cdot D Q O / 0,333+1,3 \cdot N / 0,05+2,6 \cdot P / 0,014+3 \cdot C / 0,002) / 9,9\end{array}$ \\
\hline $\begin{array}{c}\text { Navarra } \\
\text { (Ley 10/1988) }\end{array}$ & $\begin{array}{l}\mathrm{CS}_{\text {Navarra }}(€ / \text { año })=0,169 \cdot \mathrm{Q}_{\mathrm{w}} \cdot \mathrm{CC} \\
\mathrm{CC}=0,34 \cdot \mathrm{SS} / 0,286+0,55 \cdot((\mathrm{DQO}+2 \cdot \mathrm{DBO}) / 3) / 0,2677+0,11 \cdot \mathrm{N} / 0,04\end{array}$ \\
\hline $\begin{array}{c}\text { Valencia } \\
\text { (Ley 2/1992) }\end{array}$ & $\begin{array}{l}\mathrm{CS}_{\text {Valencia }}(€ / \text { año })=\left(\mathrm{TS}+0,414 \cdot \mathrm{Q}_{\mathrm{c}}\right) \cdot \mathrm{C}_{\mathrm{v}} \cdot \mathrm{C}_{\mathrm{p}} \cdot(\mathrm{CC}+\mathrm{CEC}) \\
\mathrm{TS}(€ / \text { año })=2957,17 \\
\mathrm{CC}=0,14 \cdot \mathrm{SS} / 0,3+0,14 \cdot \mathrm{DBO} / 0,3+0,18 \cdot \mathrm{DQO} / 0,5+0,07 \cdot \mathrm{N} / 0,05+0,11 \cdot \mathrm{P} / 0,02+0,11 \cdot(\mathrm{C}- \\
0,002) / 0,002+0,25 \cdot \mathrm{SI} / 3 \\
0,5<\mathrm{CC}<10\end{array}$ \\
\hline
\end{tabular}

Nota: La nomenclatura se encuentra al final del texto.

Fuente: Elaboración propia a partir de las distintas regulaciones autonómicas. 
Además de existir heterogeneidad en los parámetros de contaminación considerados en cada canon, destacamos que el precio unitario, con el que cada parámetro contribuye al cálculo de la cuota a pagar, varía ampliamente (Tabla 2). Así, los costes unitarios para las comunidades de Asturias, Aragón, Cantabria, Cataluña, Galicia y Madrid, que no refieren sus parámetros a un valor de referencia de agua urbana, son los coeficientes que acompañan a cada uno de estos parámetros en las ecuaciones de la Tabla 1, expresados explícitamente en la ley de cada una de estas comunidades. Los costes unitarios de La Rioja, Murcia, Navarra y Valencia están calculados multiplicando el tipo de cada canon por el coeficiente que acompaña a cada parámetro dividido entre el valor de referencia de cada parámetro. A modo de ejemplo, para Navarra, el coste unitario de los sólidos en suspensión se calcula multiplicando 0,169 por 0,34 y dividido entre 0,286 . En todo caso se trata de un cálculo conservador puesto que la cuota fija no se reparte entre los distintos parámetros contaminantes.

Tabla 2

Coste unitario de los parámetros considerados

\begin{tabular}{|c|c|c|c|c|c|c|c|}
\hline \multirow[b]{2}{*}{ Región } & \multicolumn{7}{|c|}{ Coste unitario $(€ / \mathbf{k g})$} \\
\hline & SS & DQO & $\mathbf{N}$ & $\mathbf{P}$ & $C\left(€ / \mathbf{S} \cdot \mathrm{cm}^{-1}\right)$ & DBO & $\begin{array}{c}\text { SI } \\
\text { (€/equitox) }\end{array}$ \\
\hline Asturias & 0,467 & 0,415 & 2,381 & 4,342 & 0,525 & - & 0,007 \\
\hline Aragón & 0,396 & 0,551 & 1,082 & - & 4,453 & - & 0,013 \\
\hline Cantabria & 0,232 & 0,268 & 0,292 & 0,585 & 3,668 & - & 0,005 \\
\hline Cataluña & 0,406 & 0,812 & 0,617 & 1,233 & 6,498 & - & 0,010 \\
\hline Galicia & 0,246 & 0,493 & 0,369 & 0,740 & 3,955 & - & 0,052 \\
\hline La Rioja & 0,439 & 0,321 & - & - & 38,792 & - & - \\
\hline Madrid & 1,100 & 1,000 & - & - & - & 1,650 & - \\
\hline Murcia & 1,145 & 0,206 & 0,893 & 6,378 & 51,515 & - & - \\
\hline Navarra & 0,201 & 0,116 & 0,396 & - & - & 0,231 & - \\
\hline Valencia & 0,193 & 0,149 & 0,577 & 2,277 & 22,770 & 0,193 & 0,035 \\
\hline
\end{tabular}

Fuente: Elaboración propia.

Dentro de los parámetros de contaminación, hacemos hincapié por su alto contenido contaminante y su difícil proceso de depuración, en la presencia de sustancias inhibitorias (SI). Se trata de contaminantes ecotóxicos especialmente nocivos para la flora y fauna y difícilmente eliminables. Generalmente en los procesos biológicos de depuración de aguas residuales (tratamientos en las EDAR) no se eliminan y pasan directamente a los ríos y mares. Por tanto, es aconsejable su eliminación mediante procesos específicos. Sin embargo, y a pesar de su nivel de contaminación, sólo son tenidos en cuenta las sustancias 
inhibitorias en seis CCAA, concretamente en Asturias, Aragón, Cantabria, Cataluña, Galicia y Valencia.

Tabla 3

Valor promedio y coeficiente de variación del coste unitario de cada parámetro de contaminación

\begin{tabular}{|l|c|c|c|c|c|c|}
\hline & SS $(\boldsymbol{\epsilon} / \mathbf{k g})$ & $\mathbf{D Q O}(\boldsymbol{\epsilon} / \mathbf{k g})$ & $\mathbf{N}(\boldsymbol{\epsilon} / \mathbf{k g})$ & $\mathbf{P}(\boldsymbol{\epsilon} / \mathbf{k g})$ & $\mathbf{C}\left(\boldsymbol{\epsilon} / \mathbf{S} \cdot \mathbf{c m}^{-1}\right)$ & $\mathbf{S I}(\boldsymbol{\epsilon} /$ equitox) \\
\hline Media & 0,48 & 0,43 & 0,83 & 2,59 & 16,52 & 0,020 \\
\hline Coef. Variación (\%) & 69,28 & 63,46 & 77,41 & 81,52 & 108,79 & 85,89 \\
\hline
\end{tabular}

Fuente: Elaboración propia.

En la Tabla 3 presentamos, a través del coeficiente de variación, la diversidad en el coste unitario de los diferentes parámetros de contaminación de las distintas estructuras de la tarifa. El coeficiente de variación está comprendido entre el 63,46\% para la demanda química de oxígeno (DQO) y el 108,79\% para la Conductividad (C), respectivamente. Este valor tan alto para la conductividad se debe principalmente al coste unitario en aquellas comunidades que normalizan los parámetros de contaminación en las ecuaciones del cálculo de la cuota a los de un agua residual doméstica y que hemos citado anteriormente. El que se normalice la ecuación a un agua residual doméstica implica que el coste del canon de saneamiento va a suponer una penalización en el importe de la cuota cuando el agua residual está más contaminada que la referencia. Además, hay algunas regiones que contribuyen más a este incremento en el coeficiente de variación que otras, tal es el caso de Madrid, donde solo se consideran tres parámetros de contaminación ( $\mathrm{SS}, \mathrm{DQO}$ y $\mathrm{DBO}$ ), con precios unitarios muy superiores a los de otras CCAA.

Con respecto al valor medio de cada parámetro de contaminación, indicamos que la conductividad (C) ofrece la mayor contribución al importe de la cuota a pagar. Seguido de los contenidos en fósforo $(\mathrm{P})$ y Nitrógeno $(\mathrm{N})$. En concordancia con los beneficios ambientales que conlleva la eliminación de estos tres tipos de contaminantes. Sin embargo, el coste unitario debido a la contaminación por sustancias inhibitorias (SI) es un orden de magnitud inferior que el correspondiente a la demanda química de oxígeno (DQO) o a los sólidos en suspensión (SS). La concentración de sustancias inhibitorias se expresa generalmente en unidades de equitox $/ \mathrm{m}^{3}$. Estas unidades de contaminación representan el número de diluciones necesarias para inmovilizar el $50 \%$ de la población del microcrustáceo Daphnia magna en 24 horas o la inhibición de la actividad luminiscente de la bacteria Photobacterium phosphoreum en 15 minutos. En ambos casos se trata de microorganismos modelo para medir toxicidad en aguas. Por ejemplo, en la Comunidad Valenciana que es el único caso 
que la presencia de SI está normalizada a un agua residual doméstica con un valor de 3 unidades de equitox $/ \mathrm{m}^{3}$, apreciamos que la toxicidad debida a sustancias inhibitorias en aguas residuales urbanas se estable en 3 unidades de equitox $/ \mathrm{m}^{3}$. Para valores superiores se penaliza la toxicidad. De acuerdo con la norma ISO 11348-3:2007, 20 unidades de equitox $/ \mathrm{m}^{3}$ se consideran tóxicas. Sin embargo, en Madrid (Ley 10/1993), o Murcia (Ley 3/2000), se permiten vertidos con hasta 25 equitox $/ \mathrm{m}^{3}$. Este hecho está en contradicción a los objetivos medioambientales que deben cumplir los cánones de saneamiento, puesto que debe evitarse que lleguen estas sustancias a los ecosistemas. Si la contribución al coste del canon por verter sustancias inhibitorias (SI) fuera mayor que la establecida en los cánones vigentes y que puede apreciarse en la Tabla 2, se incentivaría el desarrollo de nuevas tecnologías para la eliminación de estas sustancias en los procesos de tratamiento.

Estos ejemplos muestran que no existe una relación clara entre la contribución de cada parámetro de contaminación al importe del canon y sus precios sombra. De acuerdo con Molinos Senante et al., (2010), los precios sombra reflejan el valor del daño ambiental que resultaría si estos contaminantes fueran vertidos de modo descontrolado, es decir, estos precios representan una aproximación a los beneficios ambientales derivados de los procesos de tratamiento. En particular, en un estudio sobre la viabilidad económica del tratamiento de aguas residuales realizado por estos autores para 22 estaciones de depuración de aguas residuales de la Comunidad Valenciana, los precios sombra estimados para Nitrógeno $(\mathrm{N})$ y fósforo $(\mathrm{P})$, son $8,06 € / \mathrm{kg}$ y $30,94 € / \mathrm{kg}$ respectivamente, e indican el gran beneficio ambiental que produce su eliminación de los vertidos. Estos precios sombra han sido calculados para valores de contaminación típicos de depuradoras, por lo que pueden ser aplicables al presente estudio. Sin embargo, el coste unitario de los parámetros de contaminación contemplados en las ecuaciones para calcular la cuota a pagar es desproporcionalmente bajo $(0,83 € / \mathrm{kg}$ para el nitrógeno y $2,59 € / \mathrm{kg}$ para el fósforo), aun siendo dos de los parámetros con mayor peso. Para los sólidos en suspensión (SS) y la demanda química de oxígeno (DQO) la situación es la contraria, sus costes unitarios $0,48 € / \mathrm{kg}$ y $0,43 € / \mathrm{kg}$ respectivamente, son considerablemente superiores a sus precios sombra $(0,005 € / \mathrm{kg}$ y $0,131 € / \mathrm{kg}$, respectivamente). Por tanto, no existe una correlación entre el coste unitario de cada contaminante en la estructura de la tarifa de cada canon y el valor del beneficio ambiental debido a su eliminación.

\section{DESCONTAMINACIÓN DE AGUAS MEDIANTE ENERGÍA SOLAR: FOTOCATÁLISIS SOLAR}

La generación de grandes volúmenes de aguas residuales industriales (ARI) es resultado del rápido crecimiento industrial. Estas aguas tienen características 
y rangos de contaminación muy diversos, con contaminantes biodegradables, que pueden ser eliminados mediante microorganismos con tratamientos biológicos, pero también no biodegradables. Esto es debido a que frecuentemente los efluentes generados por la industria tienen una alta toxicidad y una elevada concentración de contaminante (rango de miligramos o gramos por litro de concentración). Por tanto, tienden a ser efluentes con contaminación, parcial o totalmente, no biodegradable. Por este motivo, los conocidos tratamientos biológicos no son adecuados por sí mismos para la eliminación de la contaminación presente en este tipo de aguas residuales.

Esta situación ha propiciado que en las últimas décadas se desarrollen tratamientos avanzados que sean capaces de eliminar la contaminación persistente y no biodegrable, como es el caso de los procesos de oxidación avanzada (PPOA). Éstos son tratamientos químicos en fase acuosa cuyo objetivo es generar especies altamente oxidantes (radicales hidroxilo, $\mathrm{HO}^{\circ}$, y otros radicales) que son las responsables de oxidar la materia orgánica. En los últimos 30 años, se ha investigado mucho sobre PPOA debido a la variedad de tecnologías disponibles y al amplio campo de aplicaciones. Entre los PPOA se incluyen la fotocatálisis homogénea (foto-Fenton) y heterogénea (semiconductores, fundamentalmente $\mathrm{TiO}_{2}$ ) en presencia de radiación ultravioleta (UV) o radiación visible. La posibilidad de utilizar luz solar como fuente de radiación hace a estos procesos especialmente atractivos desde el punto de vista de la sostenibilidad.

Los PPOA pueden unirse a sistemas biológicos en procesos combinados, de manera que los primeros eliminen la contaminación más resistente y tóxica, y los últimos, la biodegradable. No obstante, los PPOA presentan el inconveniente de su elevado coste frente a los tratamientos biológicos, que ya están bien establecidos como tratamientos de aguas biodegradables. Entre los PPOA destaca la fotocatálisis solar homogénea, o proceso foto-Fenton solar, que utiliza la luz del sol como fuente de energía, reduciendo los costes del suministro eléctrico del tratamiento. Aun así, el coste para depurar un efluente tóxico contaminado con $500 \mathrm{mg} / \mathrm{L}$ de un residuo de la fabricación de antibióticos (alfametil-fenilglicina) mediante foto-Fenton solar se ha estimado en $14.1 € / \mathrm{m}^{3}$ (Muñoz et al., 2008), muy superior a los $0.36 € / \mathrm{m}^{3}$ (Roman et al., 2011) que cuesta el tratamiento de agua industrial agroalimentaria biodegradable mediante un biorreactor secuencial (SBR, de sus siglas en inglés Sequencing Batch Reactor) con fangos activados. En consecuencia, se está investigando intensamente en la combinación de PPOA con tratamientos biológicos para la descontaminación de aguas residuales industriales (Oller et al., 2011) con el fin de reducir los costes del proceso y optimizar la calidad del efluente depurado. 


\section{FACTORES QUE INFLUYEN EN EL COSTE DE LOS PROCESOS COMBINADOS DE DESCONTAMINACIÓN. TRATAMIENTO SOLAR Y BIOLÓGICO}

Para ilustrar el efecto que en los costes de depuración tienen las distintas variables a considerar en el diseño del proceso de depuración, se plantea como ejemplo de la combinación de foto-Fenton solar y un reactor biológico de membrana (MBR, de sus siglas en inglés, Membrane Biological Reactor) para el tratamiento de aguas con una alta concentración de contaminantes, que dan lugar a una elevada toxicidad. El esquema de proceso sería el mostrado en la Figura 1.

Figura 1

Esquema del proceso combinado foto-Fenton y MBR

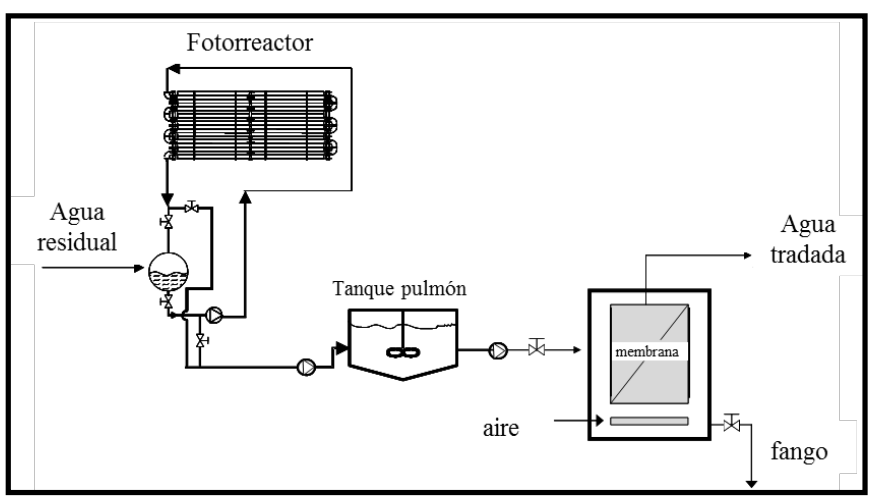

Fuente: Elaboración propia.

El proceso combinado está formado por un fotorreactor y un biorreactor de membrana, unidos mediante un tanque pulmón. El fotorreactor consiste en un lazo tubular sobre un captador curvo capaz de reflejar el 100\% de los rayos solares que llegan a su superficie hacia los tubos por los que circula el agua (captador cilíndrico compuesto, $\mathrm{CPC}$ ), dando lugar a un máximo aprovechamiento energético. La reacción de foto-Fenton genera radicales hidroxilo a partir de peróxido de hidrógeno (reactivo consumido) mediante la acción de sales de hierro en un proceso redox catalizado por la radiación solar. El MBR combina oxidación aeróbica mediante fangos activos con microfiltración tangencial a través de membranas. El agua depurada y filtrada resulta de gran calidad para su reutilización.

Al tratarse de aguas residuales con una alta toxicidad, se aplicaría un pretratamiento químico mediante foto-Fenton. Durante este tratamiento se produce la oxidación progresiva de la materia orgánica hasta $\mathrm{CO}_{2}$ (mineralización). Se 
deja que la reacción transcurra hasta que la mineralización alcanza un nivel en el que el agua ya no presenta toxicidad para los microorganismos presentes en el sistema biológico. Durante el tratamiento biológico, el resto de contaminación, que es materia orgánica biodegradable, es eliminada y se obtiene un efluente depurado. Esta estrategia de tratamiento combinado se ha probado con éxito en la depuración de aguas tóxicas (Casas et al., 2010).

En el presente trabajo, mediante experimentos en planta piloto, se ha llevado a cabo el proceso combinado descrito para tratar un agua residual industrial con una elevada concentración de sustancias inhibitorias, procedente del tratamiento de envases fitosanitarios, caracterizada por su elevado contenido en plaguicidas (Blanco et al., 2004, Moreira et al., 2012). Las características del agua residual industrial a tratar, y las del agua una vez aplicado el tratamiento combinado se muestran en la Tabla 4. Por aplicación del tratamiento descrito, se elimina la toxicidad de esta agua y se reducen los niveles de demanda química de oxígeno (DQO), demanda biológica de oxígeno (DBO), nitrógeno $(\mathrm{N})$ y fósforo $(\mathrm{P})$, por debajo de los límites de vertido.

Tabla 4

Características físico-químicas del agua residual

\begin{tabular}{|l|c|c|c|c|c|c|c|}
\hline \multicolumn{1}{|c|}{ Tipo de agua } & DBO & DQO & SS & C & N & P & SI \\
\hline Agua residual tóxica & 0,27 & 1,35 & 0,05 & 0,002 & 0,19 & 0,05 & 25 \\
\hline Agua depurada & 0,0039 & 0,013 & 0,006 & 0,002 & 0,014 & 0 & 0 \\
\hline
\end{tabular}

Nota: Resultados obtenidos experimentalmente.

Fuente: Elaboración propia.

Con respecto al coste total de este tipo de procesos combinados, a continuación comentamos los principales factores que le afectan: el tipo de reactor biológico, la concentración inicial de contaminantes y su naturaleza química, y el volumen a tratar.

\section{a) Efecto del reactor biológico utilizado}

La función del reactor biológico en el sistema combinado es eliminar la materia orgánica que queda tras el proceso foto-químico. Sin embargo, cuando se aborda desde el punto de vista económico, existe otro aspecto a considerar y es cómo contribuye el tipo de reactor biológico a acortar el tratamiento de oxidación química, que inicialmente se considera la etapa más costosa del proceso. Es decir, según la robustez del sistema biológico, éste podrá acoger aguas más o menos tratadas por el proceso químico: un tratamiento biológico muy robusto, como es el MBR, podrá depurar aguas que han sido tratadas durante menos 
tiempo por el proceso foto-químico. Así, el tiempo de tratamiento químico se acorta, reduciéndose el coste.

Figura 2

Costes totales del proceso combinado para tratar $10 \mathrm{~m}^{3} /$ día de agua tóxica industrial (DQO inicial de $1,35 \mathrm{~kg} / \mathrm{m}^{3}$ )

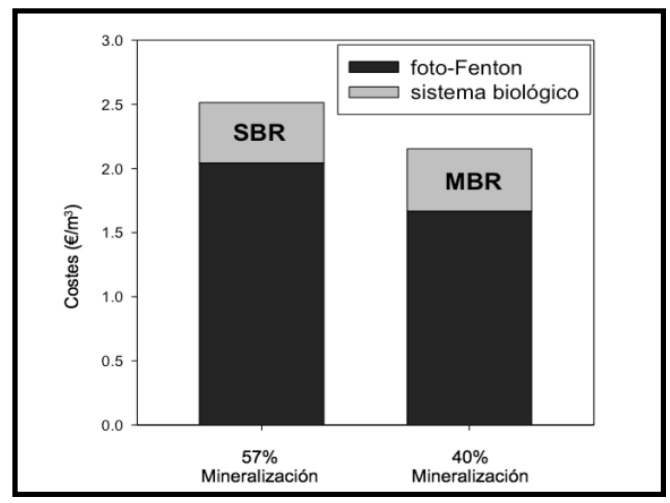

Nota: En el eje de abscisas se indica el grado de mineralización mediante foto-Fenton solar.

Fuente: Elaboración propia.

En la Figura 2, se presentan, por un lado, los costes del proceso combinado foto-Fenton con un reactor biológico secuencial (SBR, de sus siglas en inglés, Sequencing Batch Reactor) y por otro lado, foto-Fenton con MBR para tratar el agua residual tóxica descrita en la Tabla 4. Para el cálculo de estos costes se ha tenido en cuenta la inversión en reactores a través de los costes de amortización, para una vida útil de 20 años. En el primer caso, el porcentaje de mineralización (la intensidad del tratamiento químico) necesario para hacer posible el proceso de depuración biológica es del $57 \%$. Sin embargo, cuando se utiliza un MBR, sólo es necesario alcanzar el $40 \%$ de mineralización, lo que acorta el tiempo de tratamiento químico. En consecuencia, tanto los costes de operación, como los de amortización, disminuyen para el proceso combinado con MBR.

Así, los costes totales disminuyen de $2.40 € / \mathrm{m}^{3}$ para la combinación fotoFenton/SBR a $2.15 € / \mathrm{m}^{3}$ para foto-Fenton/MBR, lo que supone una reducción del $10.40 \%$. En ambos casos se aprecia que, en el proceso combinado, el mayor coste viene dado por el proceso foto-Fenton (un $85.0 \%$, cuando se combina con SBR y $77.4 \%$ del total, cuando se combina con MBR).

\section{b) Efecto de la concentración inicial de contaminante}

Como se acaba de ver, para un agua con la misma contaminación, el nivel de mineralización que es necesario obtener depende del tipo de reactor biológico. 
Pero además, la concentración inicial de contaminación afecta también a los costes. Resulta, por tanto, de interés analizar la distribución de costes de la etapa más significativa del tratamiento combinado, el proceso foto-Fenton. Estos costes son: Mantenimiento y personal, energía, reactivos químicos y amortización. Los costes de mantenimiento se pueden calcular como un $2 \%$ de los costes de amortización, los costes de personal para el tratamiento de aguas se estima en $0.036 € / \mathrm{m}^{3}$ (Molinos-Senante et al., 2010), los costes de energía se obtienen teniendo en cuenta el consumo energético de bombeo del agua en las instalaciones y los costes de amortización se hacen en base a los reactores. Para aguas con elevada carga contaminante (Tabla 4), como es el caso descrito, donde se acopla el proceso foto-Fenton con MBR, los gastos de mantenimiento y personal suponen un 2,5\%, energía el $14,8 \%$, los reactivos el $65.5 \%$ y la amortización el $17,2 \%$, respectivamente, del coste total. Con el fin de comprobar si la proporción de costes se mantiene para aguas poco contaminadas, se realizó el tratamiento en planta piloto con un agua con las mismas propiedades que las descritas en la Tabla 4, pero disminuyendo diez veces la concentración de DQO y N $\left(0,135 \mathrm{~kg} / \mathrm{m}^{3}\right.$ de DQO, $0,019 \mathrm{~kg} / \mathrm{m}^{3}$ de $\left.\mathrm{N}\right)$, que son los principales contaminantes. En este caso, la distribución de costes sería: $0.3 \%$ para mantenimiento y personal, energía el $6.8 \%$, los reactivos el $75.8 \%$ y la amortización el $13.4 \%$ del coste total. La disminución en costes de amortización es debida a la reducción en la superficie del reactor solar necesaria. Además este hecho repercute en los costes de operación, pues el coste energético $(6,8 \%)$ también disminuye al ser menor la longitud de las conducciones y por tanto la potencia para el bombeo. Los costes de mantenimiento y personal no cambian significativamente con la carga contaminante.

\section{c) Volumen tratado}

El efecto de la escala (o del volumen tratado) es muy importante en los costes. En la Figura 3 se muestra el efecto del volumen tratado sobre el proceso foto-Fenton y el MBR para el efluente con alta concentración de contaminante descrito en la Tabla 4. Partiendo de datos experimentales propios, los costes se han ido recalculando para ambos procesos variando el caudal de agua a tratar. La mayor reducción en el coste total se obtiene para capacidades de tratamiento entre 1 y $40 \mathrm{~m}^{3} /$ día, obteniendo el óptimo para caudales a partir de $100 \mathrm{~m}^{3} /$ día.

El efecto del aumento de la escala en el proceso combinado sobre los costes es mayor para el sistema biológico: el aumento de la capacidad de la planta de 1 a $40 \mathrm{~m}^{3} /$ día supone una reducción del $50 \%$ para el tratamiento biológico y del $30 \%$ para el proceso foto-Fenton para este tipo de agua. 
Figura 3

Efecto del volumen tratado sobre el coste unitario para cada etapa del proceso combinado foto-Fenton/MBR para tratar un agua industrial tóxica

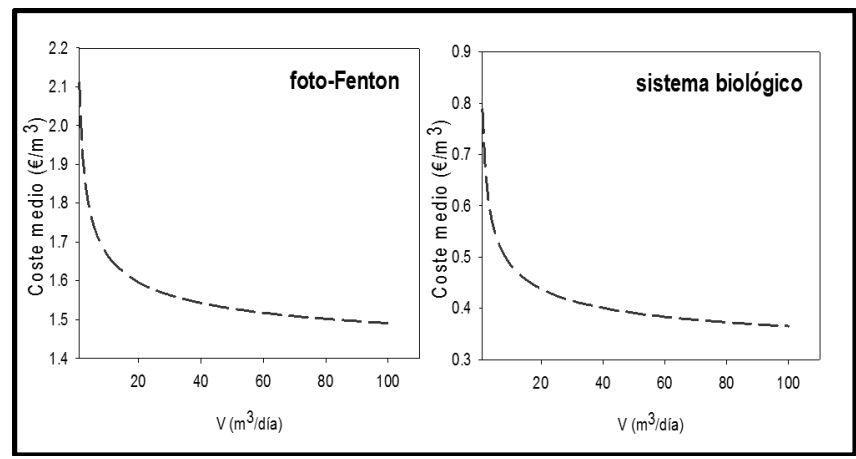

Fuente: Elaboración propia.

\section{5. ¿LA ACTUAL REGULACIÓN DE LOS VERTIDOS DE AGUAS INDUSTRIALES INCENTIVA LA INNOVACIÓN TECNOLÓGICA Y EL USO DE ENERGÍAS RENOVABLES EN EL PROCESO DE DESCONTAMINACIÓN?}

Una vez discutidos la posibilidad técnica y los costes de un proceso de descontaminación de aguas residuales industriales tóxicas mediante tecnologías que hacen uso de energías renovables, vamos a analizar el impacto que los cánones de saneamiento, como instrumentos de mercado para corregir las externalidades medioambientales negativas, tienen en el desarrollo y aplicación de estas tecnologías.

La decisión empresarial sobre invertir en tratamientos de depuración de aguas residuales antes de ser vertidas, en aras a pagar un canon de saneamiento menor, depende de la cuota del canon y del precio de la depuración. Si obtenemos un ahorro en el coste del canon superior al precio que pagamos en la depuración, invertimos en tecnologías, en caso contrario, pagaremos el canon y no nos preocuparemos de depurar las aguas.

Para las diez comunidades estudiadas aplicaremos el canon de saneamiento a un caudal de vertido de $36.500 \mathrm{~m}^{3} /$ año, correspondiente a $100 \mathrm{~m}^{3} /$ día y utilizando las propiedades físico-químicas de la Tabla 4, obteniendo así el canon de saneamiento correspondiente al agua contaminada y al agua tratada. En la Tabla 5 presentamos el importe del canon de saneamiento por cada comunidad autónoma para ese volumen de vertido y para el agua residual antes y después de ser depurada.

Mediante la aplicación del proceso combinado de foto-Fenton solar y reactor biológico de membrana, esta agua residual puede depurarse hasta alcanzar 
una DQO de salida de $0,013 \mathrm{~kg} / \mathrm{m}^{3}$ y un equitox prácticamente nulo o despreciable, a un coste total unitario de $2,153 € / \mathrm{m}^{3}$. El coste anual de depuración asciende a 78.584,5 euros, calculado teniendo en cuenta el coste unitario y el volumen total considerado. También presentamos el resultado (beneficio o pérdida) de la decisión empresarial de invertir en la tecnología propuesta (fotocatálisis solar) para descontaminar el efluente tóxico. Este beneficio o pérdida viene dado por la diferencia entre el canon a pagar por el vertido de agua residual tóxica sin tratamiento alguno, menos el canon del agua residual depurada y el coste de su depuración.

Tabla 5

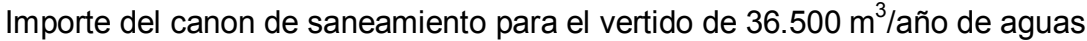
residuales tóxicas, aguas depuradas y el resultado de invertir en nuevas tecnologías de depuración, para las diez comunidades autónomas

\begin{tabular}{|c|c|c|c|}
\hline CC AA & $\begin{array}{c}\text { Canon Agua Residual } \\
\text { Tóxica } \\
(€ / a n ̃ o)\end{array}$ & $\begin{array}{c}\text { Canon Agua } \\
\text { Residual Depurada } \\
(\boldsymbol{€} / \text { año) }\end{array}$ & $\begin{array}{c}\text { Resultado (Bo/Pda) de } \\
\text { invertir en tecnologías de } \\
\text { depuración } \\
\text { (€/año) }\end{array}$ \\
\hline Aragón & $47.631,46$ & $1.420,89$ & $-32.373,93$ \\
\hline Asturias & $56.808,38$ & $5.994,64$ & $-27.770,76$ \\
\hline Cantabria & $21.205,01$ & 610,07 & $-57.989,56$ \\
\hline Cataluña & $89.717,32$ & $6.786,57$ & $4.346,25$ \\
\hline Galicia & $76.419,75$ & 795,08 & $-2.959,83$ \\
\hline La Rioja & $19.430,70$ & $4.471,25$ & $-63.625,05$ \\
\hline Madrid & $34.475,38$ & $18.630,90$ & $-62.740,03$ \\
\hline Murcia & $32.043,04$ & $4.350,33$ & $-50.891,79$ \\
\hline Navarra & $11.573,96$ & 369,36 & $-67.379,90$ \\
\hline Valencia & $58.896,21$ & $9.034,09$ & $-28.722,38$ \\
\hline
\end{tabular}

Fuente: Elaboración propia.

El valor medio del importe del canon de saneamiento para las aguas residuales sin depurar, es de 44.820,12 euros. Siendo el importe más alto el de comunidad de Cataluña, 89.717,32 euros y el más bajo el correspondiente a Navarra, 11.573,96. Estos resultados muestran la gran diferencia que existe entre los cánones de las distintas comunidades. Además, llama la atención que el valor medio del importe del canon está muy por debajo del coste total de la depuración de las aguas residuales.

Por debajo del valor medio, 44.820,12 euros, se encuentran las cuatro comunidades autónomas que no tienen en cuenta las sustancias inhibitorias (La Rioja, Madrid, Murcia y Navarra). Destacamos que el agua residual que estamos utili- 
zando como ejemplo es un agua industrial tóxica $\left(25\right.$ equitox $\left./ \mathrm{m}^{3}\right)$ y que en cuatro comunidades autónomas al no tener en cuenta en la estructura de la tarifa del canon este parámetro de toxicidad, el agente contaminador no pagará por el daño ambiental que genera. Estas aguas residuales de estas cuatro regiones irán a una EDAR que difícilmente eliminará la toxicidad que llevan, pues generalmente estas depuradoras utilizan tratamientos biodegradables que no pueden eliminar estas sustancias, por lo tanto, al final serán vertidas a ríos o mares.

En las otras seis comunidades autónomas que sí tienen en cuenta las sustancias inhibitorias en la estructura de la tarifa del canon, destacamos que todas arrojan un importe del canon de saneamiento superior al importe medio, excepto Cantabria. Esto se debe a que la estructura de la tarifa del canon de esta comunidad, contempla unos pesos relativos de los parámetros de contaminación muy bajos (Tabla 2).

En cuanto al importe del canon correspondiente al agua depurada, la diferencia entre comunidades es aún mayor, siendo, de nuevo, el importe menor el que se pagaría en Navarra, 369,36 euros. Este importe es cincuenta veces inferior al que se pagaría en la comunidad de Madrid, 18.630,90 euros. En este caso, esta diferencia en el importe del canon en las distintas comunidades no se debe a la presencia en la ecuación para calcular la cuota del parámetro de contaminación "sustancias inhibitorias", pues el agua residual ha sido tratada y dichas sustancias eliminadas. Se debe a la heterogeneidad en las distintas tarifas en cuanto a los parámetros considerados y al peso específico de cada uno de estos parámetros en las ecuaciones que permiten el cálculo de la cuota a pagar.

Finalmente, destacamos que sólo en Cataluña la inversión en depuración para el agua residual considerada supondría un beneficio. En concreto, 4.346,25 euros. Por el contrario, en las nueve comunidades restantes, sería desaconsejable desde el punto de vista empresarial invertir en esta nueva tecnología de tratamiento de aguas residuales mediante energías renovables, a pesar del importante beneficio ambiental que supone la eliminación de sustancias tóxicas del agua.

No obstante, hay que tener en cuenta que la alternativa de depuración propuesta es sostenible por el uso de la energía solar, pero no necesariamente la menos costosa. Sin embargo, la falta de información sobre de costes de tratamientos de depuración alternativos y sostenibles capaces de tratar este tipo de aguas tóxicas impiden la comparación de costes con otras técnicas.

\section{CONCLUSIONES}

La heterogeneidad en el diseño del canon de saneamiento implica marcadas diferencias tanto en el importe de la cuota a pagar como en el peso relativo de los parámetros de contaminación considerados en la estructura de la tarifa del canon. 
La decisión empresarial sobre invertir o no en depurar in situ las aguas residuales depende tanto del importe del canon como del coste de la depuración. Para que el canon de saneamiento cumpla con su objetivo de corregir el fallo de mercado de la contaminación de las aguas, en ningún caso debería ser inferior al coste de depuración.

En caso de que el canon de saneamiento sea inferior al coste de depuración por parte del empresario, éste no estará incentivado en depurar sus efluentes y los verterá a red de saneamiento, por lo que el coste de la depuración de los mismos se trasladaría al conjunto de la sociedad que financia el tratamiento público de las aguas residuales.

En particular, para el caso estudiado, al tratarse de aguas con un contenido elevado de sustancias inhibitorias que en una gran proporción no son eliminadas por los métodos convencionales implementados en las EDAR, el destino de esta contaminación será inevitablemente las aguas naturales, mares o ríos. En este sentido, existe una creciente preocupación por la cada vez mayor presencia de contaminantes persistentes en las aguas, por lo que se hace necesario el desarrollo de tecnologías sostenibles capaces de eliminar este tipo de contaminación.

En consecuencia, es necesaria la existencia de normativa medioambiental (regulación convencional o tributos medioambientales), que aumenten el nivel de exigencia con el fin de reducir progresivamente la contaminación e incentivar el desarrollo de los tratamientos de descontaminación adecuados.

\section{REFERENCIAS BIBLIOGRÁFICAS}

BEAMONTE CÓRDOBA, E., CASINO MARTíNEZ, A., VERES FERRER, E.J. (2010): "Medición de la calidad del agua mediante indicadores. Relación entre éstos y las tarifas de abastecimiento" en Estudios de Economía Aplicada, vol. 28-2, pp. (357-374)

BOLETÍN OFICIAL DE ARAGÓN, Ley 6/2001, de 17 de mayo, de ordenación y participación en la gestión de agua en Aragón, 2001, pp. 3866-3883, № 64, 01-06-2001.

BOLETÍN OFICIAL DE CANTABRIA, Ley 2/2002, de 29 de abril, de saneamiento y depuración de las aguas residuales de la comunidad autónoma de Cantabria, 2002, pp. 4275-4287, № 86, 07-05-2002.

BOLETÍN OFICIAL DEL ESTADO, Ley 10/1993, de 26 de octubre, sobre vertidos líquidos industriales al Sistema Integral de saneamiento de la Comunidad de Madrid, 1993, pp. 37578-37587, № 312, 30-12-1993. 
BOLETÍN OFICIAL DEL ESTADO, Ley 2/1992, de 26 de marzo, de saneamiento de las aguas residuales de la Comunidad Valenciana, 1992, pp. 18195-18199, № 128, 28-05-1992.

BOLETÍN OFICIAL DEL ESTADO, Ley 22/1988, de 28 de julio, de Costas, 1988, pp. 23386-23401, № 181, 29-07-1988

BOLETÍN OFICIAL DEL ESTADO, Ley 3/2000, de 12 de julio, de saneamiento y depuración de aguas residuales e implantación del canon de saneamiento de la Región de Murcia, 2001, pp. 883-891, № 8, 9-01-2001.

BOLETÍN OFICIAL DEL ESTADO, Ley $5 / 2000$, de 25 de octubre, de saneamiento y depuración de aguas residuales de La Rioja, 2000, pp. 3958839603, No 273, 14-11-2000.

BOLETÍN OFICIAL DEL ESTADO, Ley Foral 10/1988, de 29 de diciembre, de saneamiento de las aguas residuales de Navarra, 1989, pp. 3682-3684, $\mathrm{N}^{\circ}$ 32, 07-02-1989.

BOLETÍN OFICIAL DEL ESTADO, Real Decreto Legislativo 1/2001, de 20 de julio, por el que se aprueba el texto refundido de la Ley de Aguas, 2001, pp. 26791-26817, № 176, 24-07-2001

BOLETÍN OFICIAL DEL PRINCIPADO DE ASTURIAS Y DE LA PROVINCIA, Ley $1 / 1994$, de 21 de febrero, sobre abastecimiento y saneamiento de aguas en el Principado de Asturias, 1994, pp. 1141-1447, No 46, 25-021994.

BLANCO, J., MALATO, S., MALDONADO, M.I., VINCENT, M., VINCENT, J.P., SÁNCHEZ, M., MYRO, E., (2004): "The Albaida plant: first commercial step in solar detoxification", en el Global symp. on re-cycling, waste treatment and clean technology, Proceedings of REWAS 04. Madrid, September 2629, 2004. I. Gaballah, B. Mishra, R. Solozábla, M. Tanaka (Eds.). Volume II, 1425-1436. Oral presentation. Inasmet, San Sebastián, Spain 2004j.

CASAS LÓPEZ, J.L., CABRERA REINA, A., ORTEGA GÓMEZ, E., BALLESTEROS MARTÍN, M.M., SÁNCHEZ PÉREZ, A. (2010): "Integration of solar photocatalysis and membrane bioreactor for pesticides degradation" en Separation Science and Tecnology, número 45, pp. (1571-1578)

DEL VILLAR, A. (2010): "Los precios de los servicios del agua. Un análisis prospectivo de demanda sobre los usos domésticos" en Estudios de Economía Aplicada, vol. 28-2, pp. (333-356)

DIARIO OFICIAL DE GALICIA, Ley 9/2010, de 4 de noviembre, de aguas de Galicia, 2010, pp. 18886-18924, № 222, 18-11-2010.

DIARIO OFICIAL DE LA GENERALITAT DE CATALUÑA, Decreto Legislativo $3 / 2003$, de 4 de noviembre, de aguas de Cataluña, 2003, pp. 22823-22840, № 4015, 21-11-2003.

DIRECTIVA 2000/60/CE del Parlamento europeo y del Consejo, de 23 de octubre de 2000 por la que se establece un marco comunitario de actuación en le ámbito de la política de aguas. Diario Oficial de las Comunidades Europeas $L 327$ de $22-12-2000$. 
EN ISO 11348-3:2007, Calidad del agua - determinación del efecto inhibidor de muestras de agua en la emisión de luz de Vibrio fischeri (Luminiscent test de bacterias) - Parte 3: Método que utiliza bacterias liofilizadas

JIMÉNEZ, M. y LAFUENTE, R. (2007): "La conciencia ambiental: qué es y cómo medirla". IX Congreso Español de Sociología, Grupo de Trabajo 21: Sociología y Medio Ambiente, Barcelona 13-15 de septiembre de 2007

LABANDEIRA, X. (2010): "El futuro de la Imposición Ambiental" en Papeles de Economía Española, № 125/126, (Ejemplar dedicado a: Claves actuales de la fiscalidad del futuro), pp. (308-311)

MOLINOS-SENANTE, M., HERNÁNDEZ-SANCHO, F., SALA-GARRIDO, R., (2010): "Economic feasibility study for wastewater treatment: A cost-benefit analysis" en Science of the Total Environment, número 408, pp. (4396-4402)

MOREIRA, F.C., VILAR, V.J.P, FERREIRA, A.C.C., SANTOS, F.R.A., DEZOTTI, M., SOUSA, M.A., GONÇALVES, C., BOAVENTURA, R.A.R., ALPENDURADA, M.F. (2012): "Treatment of a wastewater resulting from washing of phytopharmaceutical plastic containers using combined biological and solar-driven advanced oxidation processes at pilot scale" en Chemical Engineering Journal, número 209, pp. (429-441).

MUÑOZ, I., MALATO, S., RODRIGUEZ, A., and DOMENECH, X. (2008): "Integration of environmental and economic performance of processes. Case study on advanced oxidation processes for wastewater treatment" en Journal Advanced Oxidation Technologies, número 11, pp. (270-275)

OLLER, I, MALATO, S., SÁNCHEZ-PÉREZ, J.A. (2011): "Combination of Advanced Oxidation Processes and biological treatments for wastewater decontamination - A review" en Science of the Total Environment, número 409, pp. (4141-4166)

REAL DECRETO LEGISLATIVO 1/2001, de 20 de julio, por el que se aprueba el texto refundido de la Ley de Aguas.

ROMÁN SÁNCHEZ, I.M., MOLINA RUIZ, J.M., CASAS LÓPEZ, J.L., SÁNCHEZ PÉREZ, J.A. (2011): "Effect of environmental regulation on the profitability of sustainable water use in the agro-food industry" en Desalination, número 279 , pp. (252-257) 


\section{NOMENCLATURA}

\begin{tabular}{|c|c|c|c|}
\hline$\Delta \mathrm{T}$ & Incremento de temperatura $\left({ }^{\circ} \mathrm{C}\right)$ & $\mathrm{C}_{\mathrm{w}}$ & Coeficiente de descarga (se ha considerado 1,5 ) \\
\hline$\varnothing$ & Calibre de tubería & $\mathrm{DBO}$ & Demanda biológica de oxígeno $\left(\mathrm{kg} / \mathrm{m}^{3}\right)$ \\
\hline $\mathrm{C}$ & Conductividad $(\mu \mathrm{S} / \mathrm{cm})$ & DQO & Demanda Química de Oxígeno $\left(\mathrm{kg} / \mathrm{m}^{3}\right)$ \\
\hline $\mathrm{CC}$ & Carga Contaminante & MP & Metales pesados (equimetal $/ \mathrm{m}^{3}$ ) \\
\hline $\mathrm{C}_{\mathrm{d}}$ & Coeficiente de dilución (descargas al mar) & $\mathrm{N}$ & Nitrógeno total $\left(\mathrm{kg} / \mathrm{m}^{3}\right)$ \\
\hline $\mathrm{CF}$ & Coste Fijo (€/año) & $P$ & Fósforo Total $\left(\mathrm{kg} / \mathrm{m}^{3}\right)$ \\
\hline $\mathrm{C}_{\mathrm{p}}$ & $\begin{array}{c}\text { Coeficiente pico, razón entre el máximo y la media } \\
\text { (se ha considerado 1) }\end{array}$ & $Q_{c}$ & Consumo anual de agua ( $\left.\mathrm{m}^{3} / a n ̃ o\right)$ \\
\hline $\mathrm{C}_{\mathrm{r}}$ & $\begin{array}{c}\begin{array}{c}\text { Coeficiente de reutilización; si se consume agua } \\
\text { regenerada }\end{array} \\
\end{array}$ & $Q_{w}$ & Volumen anual de vertido ( $\left.\mathrm{m}^{3} / a n ̃ o\right)$ \\
\hline $\mathrm{C}_{\mathrm{s}}$ & Coeficiente de salinidad (se ha considerado 1) & $\mathrm{SI}$ & Sustancias Inhibitorias (equitox $/ \mathrm{m}^{3}$ ) \\
\hline CS & Canon de Saneamiento (€/año) & CEC & $\begin{array}{c}\text { Coeficiente específico de contaminación (MP o } \\
\text { cambios de } \mathrm{PH} \text { ) }\end{array}$ \\
\hline $\mathrm{C}_{\mathrm{v}}$ & $\begin{array}{c}\text { Coeficiente de volumen, relación entre el caudal } \\
\text { vertido y el consumido }\end{array}$ & SS & Sólidos en Suspensión $\left(\mathrm{kg} / \mathrm{m}^{3}\right)$ \\
\hline $\mathrm{CV}$ & Coste Variable $\left(€ / \mathrm{m}^{3}\right)$ & TS & Tarifa de servicio \\
\hline
\end{tabular}

